\title{
Resistencia docente al cambio: Caracterización y estrategias para un problema no resuelto
}

\section{(Teachers resistance to change: Features and strategies for an unsolved issue)}

\author{
José Luis Córica \\ Fundación Latinoamericana para la Educación a Distancia, FLEAD (Argentina)
}

DOI: http://dx.doi.org/10.5944/ried.23.2.26578

Cómo referenciar este artículo:

Córica, J. L. (2020). Resistencia docente al cambio: Caracterización y estrategias para un problema no resuelto. RIED. Revista Iberoamericana de Educación a Distancia, 23(2), pp. 255-272. doi: http://dx.doi.org/10.5944/ried.23.2.26578

\section{Resumen}

La resistencia al cambio entendida como un comportamiento observable en respuesta al desagrado o desafío que sienten los docentes como consecuencia de la introducción de nuevas ideas, métodos o dispositivos es una constante inevitable en las organizaciones educativas. Los sistemas educativos como organizaciones dinámicas cuya misión es ofrecer respuesta a las necesidades de formación de la población han de asumir que el cambio es inevitable y, por lo tanto, lo es también la resistencia al mismo. Se requiere, por tanto, de un estudio que permita encontrar estrategias y vías de incentivo por parte de los gestores institucionales toda vez que los cambios exitosos solo son posibles cuando se ha gestionado correctamente la resistencia al mismo. El objetivo de este trabajo es realizar un estudio bibliográfico transversal que caracterice la resistencia docente al cambio, sus factores asociados, las tipologías y perfiles comunes y las estrategias posibles para enfrentarla. El resultado es un relevamiento de las distintas concepciones internacionales, los factores, las actitudes y las estrategias sugeridas por la comunidad científica. Es una problemática de larga data, irresuelta y transversal a los países, existiendo un vacío bibliográfico respecto a casos de éxito. Los resultados obtenidos permiten la formulación del marco teórico de acciones de políticas y planes estratégicos que, al contemplar la resistencia docente al cambio, mejoren las posibilidades de permeabilidad de las políticas educativas.

Palabras clave: resistencia al cambio; gestión del centro de enseñanza; política de la educación; planificación educativa. 


\begin{abstract}
Resistance to change, understood as observable behaviour in response to the displeasure or challenge teachers feel as a consequence of the introduction of new ideas, methods or devices, is a constant in educational organizations. Educational systems, as dynamic organizations willing to respond to the training needs of the population, must assume that change is inevitable and so is resistance to it. Therefore, a study is required to find out strategies and incentives for institutional managers to deal with resistance, since successful changes are only possible when resistance has been properly managed. The main goal is to develop a bibliographic study that characterizes the teaching resistance to change, its associated factors, the typologies and common profiles and the possible strategies to deal with. The result is a review of the different international conceptions, factors, attitudes and strategies suggested by the scientific community. The problem is identified as long-standing, unresolved and transversal to the countries, with a bibliographic gap regarding success stories. These results will allow the formulation of theoretical framework for educational policies and strategic plans that, when contemplating the teaching resistance to change, would improve the chances of success.
\end{abstract}

Keywords: resistance to change; school management; educational policy; educational planning.

\title{
LA RESISTENCIA HUMANA AL CAMBIO
}

Caruth y Caruth (2013) sostienen que allí donde hay cambio organizacional, existe resistencia al cambio. De acuerdo con Reilley (1989), "una ley fundamental de la física establece que a cada acción le acompaña una reacción de igual intensidad e inverso sentido" (p. 53) por lo que, ante cada propuesta de modificación del statu quo en las instituciones educativas, es de esperar naturalmente que, al menos, parte de los docentes involucrados, resistan dicho cambio. Los mismos autores señalan taxativamente que "allí donde hay cambio organizacional, habrá resistencia al cambio" (p. 12).

El estudio de la resistencia al cambio resulta entonces importante por su capacidad de proveer, a los encargados de establecer las políticas educativas, evidencias sobre cuáles son los factores involucrados, de manera tal que puedan desarrollar mejoras para conducir estas fuerzas de oposición de manera constructiva y poder avanzar (Ibrahim, Al-Kaabi y El-Zaatrani, 2013). Además, el estudio de los factores de resistencia al cambio puede ser de provecho también para los docentes, puesto que según los autores cuando un factor psicológico o personal de resistencia es identificado, los docentes pueden desarrollar una sensibilidad especial para auto detectarlos en su propia conducta, los docentes pueden cambiar sus percepciones acerca del cambio y volverse entonces más receptivos a las nuevas tendencias. 
Resulta entonces fundamental abordar el fenómeno dado que los cambios organizacionales solo serán exitosos si se trata adecuadamente la resistencia al cambio (Caruth y Caruth, 2013). Para dichos autores, esta resistencia puede ser definida como "un comportamiento observable en respuesta al desagrado o desafío que sienten los docentes como consecuencia de la introducción de nuevas ideas, métodos o dispositivos" (p. 13), o bien como "el grado en el que se está sistemáticamente en desacuerdo con cualquier iniciativa que involucre a lo nuevo dentro de la institución" (p. 13).

La resistencia al cambio en las instituciones educativas se manifiesta cuando las personas involucradas carecen de interés en el mismo, o bien tratan de disminuir su colaboración para preservar el statu quo, y consiste en acciones tanto abiertas como veladas que los docentes llevan a cabo con el fin de prevenir, interrumpir o dificultar la implementación exitosa de los cambios (Clarke, Ellett, Bateman y Rugutt, 1996).

Los sistemas educativos se materializan como organizaciones dinámicas cuya misión es dar respuesta a las necesidades de formación de la población. Siendo estas necesidades variables, han de producirse cambios en las estrategias institucionales si se quiere dar respuesta a la sociedad que se pretende servir. Al interno del sistema, los responsables de conducir la innovación han de aceptar que el cambio es inevitable y, por consiguiente, debe entenderse que la resistencia también lo es (Caruth y Caruth, 2013).

Por lo tanto, en una sociedad en la cual los cambios se suceden cada vez de manera más veloz, éstos constituyen una parte del proceso organizacional y ocurren debido a la convicción de que hay una necesidad de expandir, mejorar y transformar el sistema (Kezar, 2001). Este cambio cuya necesidad se manifiesta transversalmente en las instituciones educativas es ubicuo, continuo y ocurre a diario (Boyce, 2003), afectando todas las áreas y no simplemente aquellas que están relacionadas con las tecnologías.

Para los docentes, formados en un sistema de trabajo y acostumbrados a su propia toma de decisiones, la necesidad de ofrecer respuesta a demandas cambiantes suele resultar movilizador, ya que el cambio mueve a las personas desde lo conocido y confortable a lo desconocido, inusual e incómodo (Flamholtz y Randle, 2008) produciendo una diversidad de temores e incertidumbres, por lo que la resistencia al cambio es, en el fondo, la resistencia a la idea de perder algo que es valioso o perder algo conocido para ganar algo desconocido (Ibrahim, Al-Kaabi y El-Zaatrani, 2013).

Por tanto, si la realidad social es dinámica, la velocidad de los cambios es creciente y el sistema educativo en su conjunto debe asumir como misión dar respuesta a las necesidades formativas de la sociedad en la que se encuentra inmerso; el cambio resulta tan inevitable como la resistencia de los docentes a éste. Han de encontrarse entonces estrategias y vías de incentivo para hacer que este cambio sea posible. A este respecto, Palmer, Dunford y Akin (2009) encontraron que no todas las personas de la organización lo resisten y que existen también incentivos para motivarlo, como la seguridad, la remuneración, la autoridad, el estatus, la responsabilidad, las 
condiciones de trabajo, la auto satisfacción y la disponibilidad de tiempo y esfuerzo necesarios. Serán éstas las áreas potenciales a las que los gestores del cambio deberán prestar especial atención, toda vez que el progreso de las transformaciones en las instituciones educativas está afectado, hasta un cierto grado, por las actitudes de resistencia que prevalecen en los cuerpos docentes (Ibrahim, Al-Kaabi y El-Zaatrani, 2013).

\section{MÉTODO}

El presente trabajo constituye una operación documental con el fin de recuperar y analizar un conjunto de documentos o referencias bibliográficas que se han publicado sobre esta temática específica y se entiende como un estudio detallado, selectivo y crítico que integra la información esencial de una perspectiva (Girao, 2015).

Se siguió los lineamientos de Merino-Trujillo (2011) pretendiendo como finalidad resumir la información sobre la temática, identificar los aspectos relevantes, identificar las variables asociadas al estudio del tema, proporcionar información amplia para ahorrar tiempo y esfuerzo en la lectura de documentos primarios y ayudar al lector a preparar comunicaciones, contribuyendo a superar las barreras idiomáticas y discutiendo las conclusiones.

Siguiendo a Cué et al. (2008), se conformaron los siguientes pasos: definición del tema, elaboración del plan de trabajo, búsqueda bibliográfica, selección y acceso a los documentos, análisis y síntesis de la información y redacción del artículo.

\section{ORIGEN Y NATURALEZA DE LA RESISTENCIA DOCENTE AL CAMBIO}

Nada produce tanta ansiedad en el lugar de trabajo que la frase "el cambio viene" (Lane, 2007) (p. 85). En los cuerpos profesorales, la incertidumbre es uno de los factores principales de resistencia, "en especial para aquellos individuos con insuficiente capacidad de afrontamiento del cambio" (Caruth y Caruth, 2013) (p. 12). Dado que la naturaleza de la resistencia al cambio es subjetiva (Ford y Ford, 2010), a pesar del hecho de que los cambios se implementen por razones positivas, los miembros de la organización frecuentemente reaccionan en forma negativa y resistente (Boohene y Williams, 2012) aun cuando éste sea implementado por razones loables como la adaptación a las condiciones ambientales o permanecer competitivos (Kreitner y Kinicki, 2010). Esto contribuye a que la inclusión de TIC en el sistema educativo sea aún muy pobre (Padilla, 2018).

La resistencia al cambio suele tener origen en la sensación de que éste es requerido como remedio a un mal desempeño profesional en las actividades que la institución educativa en general y los profesores en particular, están realizando de manera cotidiana. La sola idea crea inestabilidad y amenaza a las instituciones educativas, ya que es percibido como una prueba de que la estructura actual es 
defectuosa o inadecuada, y tal percepción puede generar reacciones defensivas que se suman a los sentimientos de angustia y fracaso (Caruth y Caruth, 2013).

Complementariamente, si además de inferir que el cambio tiene origen en deficiencias a corregir en las prácticas áulicas, los docentes sienten que las nuevas propuestas amenazan su identidad profesional, la resistencia se agrava. Los mismos autores sostienen que "la forma en la que los docentes reaccionan a las reformas educativas está determinada en gran medida por la percepción que los mismos tengan respecto de si sus identidades profesionales están siendo reforzadas o amenazadas por los cambios propuestos" (p. 109).

\section{Resistencia al modo de implantación}

Para Palmer et al. (2009), los docentes resisten cuando detectan errores en el modo de implementación (factor organizacional) en especial si han pasado por frecuentes cambios y contracambios en un período limitado de tiempo o si han sido testigos de los efectos negativos de procesos de transformación anteriores.

Resulta fundamental el nivel de credibilidad en las habilidades y competencias del equipo que lidera la implantación para despertar la confianza de los docentes en dicho proceso. Al respecto, Caruth y Caruth (2013) señalan que la falta de confianza en quienes conducen el cambio puede pronosticar reacciones de descreimiento que luego llevan a una resistencia intencionada a los cambios organizacionales: "El descreimiento como resultado del cambio organizacional tiene gran impacto en la intención de resistir el cambio por parte de los trabajadores" (p. 16).

En el mismo sentido opinan Ibrahim, Al-Kaabi y El-Zaatrani (2013) cuando sostienen que la resistencia al cambio se ve reforzada cuando éstos son introducidos sin el debido proceso de planeamiento, cuando los docentes no fueron preparados con anticipación, y cuando éstos sienten que las transformaciones son impuestas, antes que surgidas de la realidad cotidiana de sus trabajos. También Caruth y Caruth (2013) afirman que "la resistencia al cambio de los docentes tiene especial relación a la forma en la que los cambios son introducidos".

Debido a la naturaleza compleja y subjetiva de la resistencia al cambio, Ford y Ford (2009a) sugieren una aproximación "conversacional" en los casos en que se perciba resistencia, con el fin de identificar por qué los gestores del cambio llaman como resistente a una actitud en particular, tomando un modelo de aproximación constructivista que ve, en las manifestaciones de resistencia al cambio por parte de los docentes, una oportunidad de aprendizaje, de entender y mejorar el proceso de transformación, y de crecimiento por parte de los gestores responsables de implementar el mismo (Snyder, 2017).

Aun así, no siempre es simple identificar la resistencia al cambio, puesto que en ocasiones sucede que la oposición a una iniciativa tiene origen en que la misma es considerada inadecuada por el profesorado de una comunidad concreta. Al respecto, Ford y Ford (2009b) plantean que las razones, los pensamientos y emociones que 
acompañan a la resistencia al cambio son de naturaleza compleja. No todos los comportamientos resistentes han de ser vistos como resistencia sistemática, dado que en muchas oportunidades el origen es un esfuerzo legítimo para mantener los objetivos institucionales, en especial por parte de los miembros con mayor historia institucional y compromiso.

\section{Resistencia originada en las tecnologías}

En particular, una de las formas de integración de tecnologías que se ha convertido en los últimos tiempos en el ejemplo icónico del cambio en la educación superior es la educación en línea, puesto que la transformación en el sistema de enseñanza -aprendizaje y sus prácticas son frecuentemente resistidos por los docentes y en el caso de la educación que cambia la relación cara a cara entre docente y estudiantes, la oposición ha sido abierta y explícita (Caruth y Caruth, 2013).

$\mathrm{Al}$ respecto de la introducción de cambios en los métodos de enseñanza, AlAteeqi (2009) indica que, aun cuando las metodologías interactivas promueven la creatividad y la innovación en la enseñanza, algunos docentes prefieren los métodos y estilos antiguos. Esto crea un conflicto entre las autoridades y los encargados de establecer las políticas educativas. Los argumentos frecuentes para la resistencia a la innovación que implica la educación en línea son descritos por Folkers (2005) quien señala que, entre las razones que se esgrimen para el cambio hacia la virtualidad, se encuentran la calidad de la educación en línea, los desafíos para evaluar el proceso de los estudiantes, la carga creciente de trabajo, los derechos de propiedad intelectual de cursos y recursos, entre otros.

Sin embargo, Lane (2007) señala que la resistencia al cambio de las instituciones educativas en lo que a la implementación de educación en línea respecta, son principalmente resultado inevitable de la incertidumbre.

\section{FACTORES ASOCIADOS A LA RESISTENCIA DOCENTE AL CAMBIO}

La resistencia al cambio docente demora y ralentiza cualquier proceso de reforma educativa y puede tomar dos formas bien definidas: abierta o encubierta. La resistencia abierta se manifiesta en acciones que pueden ser productivas si se manejan de manera positiva, mientras que las encubiertas son más complejas de manejar porque incluyen la obstrucción o la obstaculización de los esfuerzos de cambio (Snyder, 2017). Por su parte, Padilla (2018) señala las percepciones negativas con respecto a las TIC como factor relevante, agrupando la resistencia en dos tipologías: causas relacionadas con los aspectos técnicos o barreras y causas relacionadas con los aspectos pedagógicos. Respecto de las primeras, Jhonson (2018) demuestra que una actitud positiva respecto de los cambios permite superar las barreras percibidas.

Los factores de la resistencia al cambio por parte de los docentes han sido abordados desde distintas facetas que incluyen los asociados a la personalidad, 
aquellos que se agrupan en torno a las características de la cultura institucional y los que se encuentran relacionados con la auto percepción de los docentes respecto de su propia autoeficacia en el uso de las tecnologías.

\section{Factores asociados a la personalidad}

Ibrahim, Al-Kaabi y El-Zaatrani (2013) describen tres tipos de factores asociados a la resistencia al cambio: los factores psicológicos, los factores personales y los asociados a la falta de confianza. Para los autores, los factores psicológicos influyen de modo determinante en la resistencia al cambio por parte de los docentes. Ejemplos de estos factores son las sensaciones de perdida, amenaza, duda, incomodidad o preocupaciones. Por su parte, los factores personales de la resistencia al cambio están asociados a la identidad, las actitudes, las creencias, la adaptabilidad y la confianza. También sobre factores personales se manifiestan Graetz, Rimmer, Lawrence y Smith (2002), señalando como factor personal relevante que los docentes consideren que el cambio a ser implementado es innecesario (si las cosas están bien, para qué cambiarlas).

Finalmente, la falta de confianza, las tendencias conservadoras relacionadas con la edad, las diferentes percepciones de los peligros externos o el desacuerdo respecto de las estrategias propuestas para su materialización, son factores que también contribuyen a la resistencia docente. Por su parte, Palmer et al. (2009) agregan como factor a la incomodidad y la incertidumbre.

\section{Factores asociados a la cultura institucional}

Per (2004) señala la existencia de otro grupo de factores, relacionados con la cultura institucional, ya que ésta tiene un efecto fundamental en las actitudes de adopción o resistencia, explicando que dichos factores incluyen el modo en que las normas y los valores toman forma, cómo el trabajo es institucionalmente organizado, cómo se crean las relaciones interpersonales y cómo la misma idea del cambio y la renovación es interpretada en la institución educativa.

Un caso especial de cultura institucional es el de las instituciones de educación superior en general y las universitarias en particular, cuya inercia para la adopción de los cambios es explícitamente mencionada por Caruth y Caruth (2013), cuando nos dicen que "para decirlo de manera sencilla, las universidades han sido criticadas históricamente por ser demasiado lentas para implementar los cambios" (p. 13). Para los autores, la implementación de cambios educativos en los sistemas universitarios seguirá siendo un desafío porque las universidades son lentas en la implementación de los mismos, debido a que tratan de mantener un balance entre tradición e innovación. En el mismo sentido se manifiestan Keenan y Marchel (2007).

En las instituciones universitarias, el proceso de socialización por el que las personas adquieren identidad, heredan y transmiten las normas, el comportamiento, 
las costumbres y las ideologías, es naturalmente resistente a los cambios organizacionales. De hecho, los profesores universitarios pueden llegar incluso tan lejos como argumentar que su resistencia al cambio se debe a que éstos limitan su libertad de cátedra o libertad académica cuando, de hecho, son los profesores y no las autoridades los que, en general, controlan las características fundamentales de las prácticas docentes en las instituciones de educación superior (Caruth y Caruth, 2013).

Sin embargo, no todos los profesores resisten por igual. Clarke et al. (1996) sostienen que las investigaciones muestran que los profesores más antiguos y, en especial, los varones con propiedad sobre las cátedras y los profesores de tiempo completo son los que tienen más probabilidades de presentar resistencia al cambio.

\section{Factores asociados a la percepción de autoeficacia}

De entre los factores de resistencia al cambio analizados por los diversos autores, se describe especialmente la autoeficacia percibida por parte del docente, misma que se divide en dos factores. El primero es la eficacia personal, o la percepción de que se poseen las habilidades necesarias para llevar adelante el cambio (Ashton y Webb, 1986). La segunda es la eficacia docente, o la creencia de que los estudiantes se beneficiarán a partir de los cambios producidos (Gibson y Dembo, 1984).

Por su parte, Araya y Orellana (2018) destacan la importancia de la barrera tecnológica auto percibida, así como el desconocimiento de las herramientas y de las metodologías como causa principal de la resistencia docente. Otro factor que puede influenciar la respuesta de los docentes a la innovación es el grado de cansancio crónico, descrito como síndrome de Burnout. El cansancio crónico puede ser pensado como una de las muchas posibles variables moderadoras que afectan los valores de la expectativa de desempeño y el valor subjetivo asignado a las iniciativas (Gonsiewski, 2014).

El autor sostiene que las investigaciones que estudian la relación entre el cansancio crónico y la autoeficacia sindican que ambas variables están correlacionadas negativamente. Así, los docentes con una alta autoeficacia percibida tienen una probabilidad menor de experimentar cansancio crónico, mientras aquellos que no se auto perciben como eficaces, tienden a mostrar mayores niveles de agotamiento (Evers, Brouwers, y Tomic, 2002; Bümen, 2010; Brouwers, Tomic, y Boluijt, 2011; Brown, 2012). Si bien la relación entre autoeficacia percibida y cansancio crónico ha sido identificada consistentemente como correlación fuerte, la relación entre aquella y la aceptación del cambio ha sido menos establecida.

Estos factores han sido descritos recientemente a través de un modelo matemático de factores explicativos de la resistencia al cambio y la incorporación de TIC (Córica, 2019) en el ámbito particular de los docentes de Argentina. 


\section{ESTRATEGIAS POSIBLES PARA ENFRENTAR LA RESISTENCIA DOCENTE AL CAMBIO}

Los encargados de la implantación de los procesos de cambio han de desarrollar diversas estrategias para gestionar las reacciones de resistencia de las personas (Yllmaz y Kılıçoğlu, 2013). La cuestión central respecto a la resistencia al cambio consiste en orientar las fuerzas del cambio organizacional que causan la resistencia para poder reducirlas, ya que las razones por las cuales muchas organizaciones fallan en llevar a cabo dichas iniciativas están asociadas con una subestimación de la influencia del cambio sobre los individuos (Kavanagh y Ashkanasy, 2006).

Resulta fundamental, por tanto, tomar conciencia de que la gestión del cambio se basa en el entendimiento del comportamiento humano en las organizaciones toda vez que, como consecuencia de los desafíos que el mismo plantea, las personas pueden reaccionar con emociones como la incertidumbre, frustración, temor y sentirse amenazadas y desorientadas y, en consecuencia, exhibir una actitud defensiva y negativa respecto del cambio (Yılmaz y Kılıçoğlu, 2013). Puede concluirse por tanto que, si los docentes observan que las transformaciones propuestas están en concordancia con sus actitudes y creencias, y son menos amenazantes para ellos, es mucho más probable que no manifiesten resistencia. Por el contrario, mucha de la resistencia actualmente percibida es consecuencia de la falta de alineación de las directivas de cambio con los valores o metas de los docentes (D’Elía, 2019).

El debate actual ya no es si las instituciones de educación superior deben o no cambiar. La pregunta sobresaliente es cómo deben llevarse a cabo los cambios para transformarse y adaptarse a los desafíos actuales (Caruth y Caruth, 2013). Si bien las sugerencias y recomendaciones difieren, todas las propuestas sostienen que se requieren acciones profundas para crear un proceso más funcional de gobernanza que pueda afrontar los cambios del presente y del futuro (Kezar, 2005), aun en un ambiente donde la gestión de cambio es multifacética y compleja, puesto que a los académicos no puede simplemente decírseles lo que tienen que hacer (Grant, 2003) y la persuasión de los profesores en estas condiciones resulta un proceso complejo (Caruth y Caruth, 2013).

A pesar de los pronósticos esperanzadores de los diseñadores de política educativa y de las autoridades, la implementación efectiva continúa siendo inconsistente (Tyack y Cuban, 1995; Payne y Kaba, 2007; Dufour y Marzano, 2011). Campos y Ramírez (2018) sostienen, por su parte, que no logra hoy superarse la dicotomía entre grupos de apoyo y de resistencia originada en la falta de coherencia de las políticas educativas.

La planificación y la programación del proceso resulta fundamental, considerando que la cantidad de tiempo disponible y el entusiasmo por parte de los docentes para llevar a cabo los cambios, impactará en las sensaciones de resistencia (Lane, 2007). Para evitar el descreimiento, los líderes deberán proveer de manera oportuna la información completa respecto a las transformaciones que se intentan realizar. La 
calidad de esta información es el factor de mayor efecto en el descreimiento ante el cambio (Caruth y Caruth, 2013) y, tal como señalan Constantinescu y Alexandrache (2014), la aceptación de las reformas o la resistencia a los cambios está fuertemente ligada con el grado de involucramiento docente.

\section{TABLAS DEL ANÁLISIS BIBLIOGRÁFICO: CONCEPTO, FACTORES, ACTITUDES Y ESTRATEGIAS}

A continuación, se muestra en la tabla 1 las diversas concepciones del cambio y su resistencia encontradas en la literatura.

Tabla 1. Concepción del cambio y su resistencia según los autores

\section{Concepción del cambio y su resistencia según los autores}

La resistencia al cambio es un

comportamiento observable en respuesta al

desagrado o desafío que sienten los docentes Caruth y Caruth, (2013)

como consecuencia de la introducción de

nuevas ideas, métodos o dispositivos.

La resistencia al cambio es el grado en el que se está sistemáticamente en desacuerdo con cualquier iniciativa que involucre a lo nuevo dentro de la institución.

El cambio es una parte del proceso organizacional y ocurre debido a la convicción de que hay una necesidad de expandir, mejorar y transformar el sistema.

El cambio mueve a las personas desde lo conocido y confortable a lo desconocido, inusual e incómodo.

La resistencia al cambio es, en el fondo, la resistencia a la idea de perder algo que es valioso o perder algo conocido para ganar algo desconocido.

La resistencia al cambio es un mecanismo de adaptación a las condiciones ambientales o para permanecer competitivos.

La resistencia al cambio es una oportunidad de aprendizaje, de entender y mejorar el proceso de cambio, de crecimiento y aprendizaje por parte de los gestores responsables de implementar.

Caruth y Caruth, (2013)

Kezar, (2001)

Flamholtz y Randle, (2008)

Ibrahim, Al-Kaabi y El-Zaatrani, (2013)

Kreitner y Kinicki, (2010) 
Tabla 2. Principales factores que influyen en la resistencia al cambio según los autores

\section{Principales factores que influyen en la resistencia al cambio}

\begin{tabular}{|c|c|}
\hline Sensación de amenaza & $\begin{array}{l}\text { Caruth y Caruth, (2013. } \\
\text { Ibrahim, Al-Kaabi y El-Zaatrani, (2013) }\end{array}$ \\
\hline Ansiedad & $\begin{array}{l}\text { Yılmaz y G Kılıçoğlu, (2013) } \\
\text { Gonsiewski, (2014) }\end{array}$ \\
\hline Avance de las TIC & $\begin{array}{l}\text { Folkers, (2005) } \\
\text { Lane, (2007) }\end{array}$ \\
\hline Agotamiento crónico (síndrome de burnout) & $\begin{array}{l}\text { Gonsiewski, (2014) } \\
\text { Brouwers, Tomic y Boluijt, (2011) } \\
\text { Brown, (2012) } \\
\text { Bümen, (2010) } \\
\text { Evers, Brouwers y Tomic, (2002) }\end{array}$ \\
\hline Falta de confianza en el equipo implantador & $\begin{array}{l}\text { Ibrahim, Al-Kaabi y El-Zaatrani, (2013) } \\
\text { Caruth y Caruth, (2013) }\end{array}$ \\
\hline Conflicto & $\begin{array}{l}\text { Per, (2004) } \\
\text { Kezar y Eckel, (2002) } \\
\text { Al-Ateeqi, (2009) }\end{array}$ \\
\hline Cultura institucional & $\begin{array}{l}\text { Caruth y Caruth, (2013) } \\
\text { Yllmaz y Kılıçoğlu, (2013) } \\
\text { Palmer et al. (2009) } \\
\text { McBride, (2010) }\end{array}$ \\
\hline Incertidumbre & $\begin{array}{l}\text { Ibrahim, Al-Kaabi y El-Zaatrani, (2013) } \\
\text { Caruth y Caruth, (2013) } \\
\text { Lane, (2007) } \\
\text { Palmer et al. (2009) } \\
\text { Yllmaz y Kılıçoğlu, (2013) } \\
\end{array}$ \\
\hline Incomodidad & $\begin{array}{l}\text { Ibrahim, Al-Kaabi y El-Zaatrani, (2013) } \\
\text { Palmer et al. (2009) }\end{array}$ \\
\hline Innecesidad del cambio & $\begin{array}{l}\text { Graetz, Rimmer, Lawrence y Smith, (2002) } \\
\text { Yılmaz y Kılıçoğlu, (2013) }\end{array}$ \\
\hline Modo de implantación & $\begin{array}{l}\text { Palmer et al. (2009) } \\
\text { Flamholtz y Randle, (2008) } \\
\text { Yllmaz y Kılıçoğlu (2013) } \\
\text { Ibrahim, Al-Kaabi y El-Zaatrani, (2013) } \\
\text { Caruth y Caruth, (2013) }\end{array}$ \\
\hline Percepción de baja autoeficacia & $\begin{array}{l}\text { Ashton y Webb, (1986) } \\
\text { Caruth y Caruth, (2013) }\end{array}$ \\
\hline $\begin{array}{l}\text { Temor a una o más de estas pérdidas: de } \\
\text { contacto, de control, de creatividad, de } \\
\text { habilidades, de independencia de influencia, } \\
\text { de satisfacción laboral, de poder, de status, } \\
\text { de seguridad. }\end{array}$ & $\begin{array}{l}\text { Snyder, (2017) } \\
\text { Yılmaz y Kılıçoğlu, (2013) }\end{array}$ \\
\hline
\end{tabular}


Tabla 3. Actitudes en las que se manifiesta la resistencia al cambio según los autores

\section{Actitudes en las que se manifiesta la resistencia al cambio según los autores}

\begin{tabular}{|l|l|}
\hline Colocarse a la defensiva & Yllmaz y Kllı̧̧ŏlu, (2013) \\
\hline Mantener el statu quo & $\begin{array}{l}\text { Hargreaves, (2005) } \\
\text { Snyder, (2017) }\end{array}$ \\
\hline $\begin{array}{l}\text { Escepticismo generalizado sobre las } \\
\text { posibilidades reales de que el cambio se } \\
\text { produzca }\end{array}$ & $\begin{array}{l}\text { Ibrahim, Al-Kaabi y El-Zaatrani, (2013) } \\
\text { Hargreaves, (2005) } \\
\text { Huberman, (1988) }\end{array}$ \\
\hline Asumir una actitud negativa & Yllmaz y Kllıçŏlu, (2013) \\
\hline $\begin{array}{l}\text { Crear o sostener conflicto con los gestores } \\
\text { del cambio }\end{array}$ & Caruth y Caruth, (2013) \\
\hline Disminuir la colaboración & Clarke, Ellett, Bateman y Rugutt, (1996) \\
\hline $\begin{array}{l}\text { Oposición abierta y explicita a las iniciativas } \\
\text { de cambio }\end{array}$ & Caruth y Caruth, (2013) \\
\hline $\begin{array}{l}\text { Oposición encubierta y/o sabotaje de las } \\
\text { iniciativas }\end{array}$ & $\begin{array}{l}\text { Snyder, (2017) } \\
\text { Kreitner y Kinicki, (2010) }\end{array}$ \\
\hline Resistencia intencionada & Caruth y Caruth, (2013) \\
\hline Resignación pasiva & Kreitner y Kinicki, (2010) \\
\hline
\end{tabular}

Tabla 4. Estrategias posibles para la resistencia al cambio según los autores

\section{Estrategias posibles para la resistencia al cambio según los autores}

\begin{tabular}{|l|l|}
\hline $\begin{array}{l}\text { Afrontar los cambios que resultan de la } \\
\text { implementación de TIC. }\end{array}$ & Kezar, (2005) \\
\hline $\begin{array}{l}\text { Asumir la motivación del docente como } \\
\text { responsabilidad de quienes lideran el } \\
\text { cambio. }\end{array}$ & McBride, (2010) \\
\hline $\begin{array}{l}\text { Brindar atención a las preocupaciones de los } \\
\text { docentes. }\end{array}$ & Ibrahim, Al-Kaabi y El-Zaatrani, (2013) \\
\hline Capacitar a los docentes para el cambio. & Ibrahim, Al-Kaabi y El-Zaatrani, (2013) \\
\hline $\begin{array}{l}\text { Comunicar con claridad la inminencia del } \\
\text { cambio. }\end{array}$ & Hartley, (2009) \\
\hline $\begin{array}{l}\text { Considerar en la estrategia de } \\
\text { implementación, los factores de resistencia } \\
\text { al cambio relacionados con la cultura } \\
\text { institucional. }\end{array}$ & Per, (2004) \\
\hline $\begin{array}{l}\text { Crear en el cuerpo profesoral la conciencia } \\
\text { del cambio. }\end{array}$ & Ibrahim, Al-Kaabi y El-Zaatrani, (2013) \\
\hline $\begin{array}{l}\text { En el proceso de implantación, no ignorar o } \\
\text { dejar sin atención la resistencia al cambio. }\end{array}$ & McBride, (2010) \\
\hline
\end{tabular}




\section{Estrategias posibles para la resistencia al cambio según los autores}

\begin{tabular}{|c|c|}
\hline Dar apoyo e incentivar la motivación & Ibrahim, Al-Kaabi y El-Zaatrani, (2013) \\
\hline para reducir la sensación de ansiedad y & DuFour, DuFour y Eaker, (2008) \\
\hline frustración de los docentes ante el cambio. & Rayan y Ackerman, (2005) \\
\hline Desarrollar la confianza mutua. & $\begin{array}{l}\text { Beckun, (2010) } \\
\text { McGregor, (2002) }\end{array}$ \\
\hline $\begin{array}{l}\text { Evitar el reducir la estrategia a decirle a los } \\
\text { docentes lo que tienen que hacer. }\end{array}$ & Grant, (2003) \\
\hline $\begin{array}{l}\text { Garantizar las oportunidades de interacción } \\
\text { entre los docentes involucrados en el } \\
\text { cambio. }\end{array}$ & Hartley, (2009) \\
\hline $\begin{array}{l}\text { Gestionar como factores claves la } \\
\text { educación, la comunicación, la participación } \\
\text { involucrante, la facilitación y apoyo, la } \\
\text { negociación y el acuerdo, la manipulación } \\
\text { y cooptación, y la coerción explícita o } \\
\text { implícita. }\end{array}$ & Kotter y Schlesinger, (1979) \\
\hline Mantener a los profesores informados. & Ibrahim, Al-Kaabi y El-Zaatrani, (2013) \\
\hline $\begin{array}{l}\text { Modelar los comportamientos para poder } \\
\text { establecer una estrategia correcta. }\end{array}$ & DuFour, DuFour y Eaker, (2008) \\
\hline $\begin{array}{l}\text { No descuidar las percepciones psicológicas } \\
\text { de las personas respecto del cambio } \\
\text { y conducir con eficacia los factores } \\
\text { psicológicos del proceso. }\end{array}$ & $\begin{array}{l}\text { Devos, Buelens y Bouckenooghe, (2007) } \\
\text { Armenakis y Bedeian, (1999) } \\
\text { Martin, Jones y Callan, (2005) }\end{array}$ \\
\hline $\begin{array}{l}\text { No subestimar la influencia del cambio sobre } \\
\text { los individuos. }\end{array}$ & Kavanagh y Ashkanasy, (2006) \\
\hline $\begin{array}{l}\text { Orientar las fuerzas del cambio } \\
\text { organizacional que causan la resistencia para } \\
\text { poder reducirlas. }\end{array}$ & Yılmaz y Kılıçoğlu, (2013) \\
\hline $\begin{array}{l}\text { Planificar y programar cuidadosamente la } \\
\text { propuesta de cambio. }\end{array}$ & Lane, (2007) \\
\hline $\begin{array}{l}\text { Poseer en la estrategia de implementación } \\
\text { un líder eficiente cuyos comportamientos } \\
\text { se originen a partir de valores morales } \\
\text { y creencias que sean congruentes con el } \\
\text { cambio propuesto. }\end{array}$ & Silcox, MacNeill y Cavanagh, (2003) \\
\hline $\begin{array}{l}\text { Proveer de manera oportuna la información } \\
\text { completa y de calidad respecto del cambio } \\
\text { que se intenta realizar. }\end{array}$ & Caruth y Caruth, (2013). \\
\hline $\begin{array}{l}\text { Realizar ingentes esfuerzos para crear una } \\
\text { sensación de comunidad que involucre a los } \\
\text { docentes. }\end{array}$ & $\begin{array}{l}\text { Hartley, (2009) } \\
\text { Ibrahim, Al-Kaabi y El-Zaatrani, (2013) }\end{array}$ \\
\hline
\end{tabular}


El análisis realizado muestra con claridad que, si bien la resistencia al cambio es un fenómeno multicausal, el grueso de los autores coincide en que la misma está fuertemente relacionada con factores personales (especialmente los temores y la incertidumbre), siendo también factores relevantes los institucionales, la autopercepción de baja autoeficacia y la resistencia a los modos de implantación.

\section{CONCLUSIONES}

La resistencia al cambio por parte de los docentes es un problema transversal a culturas y países que continúa irresuelto luego de décadas de investigación. Existe un importante vacío bibliográfico de experiencias reales de éxito, así como de políticas educativas que hayan mostrado resultados sistemáticos al respecto tanto a nivel general como a nivel de resistencia a la incorporación de TIC. Los principales factores de resistencia están relacionados con factores asociados a la personalidad, a la cultura institucional y a la percepción de auto eficacia de los docentes.

El cambio en educación es inevitable, como así también lo es enfrentar procesos de resistencia al mismo. Los cambios institucionales exitosos solo son posibles cuando se ha gestionado correctamente la resistencia al cambio (Caruth y Caruth, 2013). Las autoridades llevan décadas tratando de integrar las TIC al sistema educativo habiendo obtenido a la fecha escasos niveles de éxito (Ryals, 2019) por la dificultad encontrada para lograr una actitud positiva respecto de la brecha digital, la falta de credibilidad docente en las capacitaciones (Molina, Mesa y Fredy, 2018) y la existencia de liderazgos cerrados y verticalistas (D'Elía, 2019).

Sin importar cuan efectivo sea el cambio, los profesores y el resto de los miembros de la comunidad deben estar motivados para innovar. La creación de esa clase de motivación "es responsabilidad de quienes lideran" (McBride, 2010) (p. 6). Para que una organización supere la resistencia al cambio, los involucrados de todos los niveles deben quedar involucrados en el proceso.

\section{REFERENCIAS}

Al-Ateeqi, A. (2009). We have come along way: Redefining education and its global challenges in the United Arab Emirates. Education without Borders Conference. Congreso llevado a cabo en Emiratos Árabes Unidos.

Araya, S. V., y Orellana, R. X. (2018). Representaciones de docentes universitarios respecto de las TIC en la acción práctica: Algunas claves para el diseño de instancias formativas. REXE. Revista de Estudios y Experiencias en Educación, 2. doi: 10.21703/rexe. Especial3201845584

Armenakis, A., y Bedeian, A. (1999). Organizational change: A review of theory and research in the 1990s. Journal of Management, 2(3), 293-315.

Ashton, P. T., Webb, R. B. (1986). Making a difference: Teachers' sense of efficacy and student achievement. Longman.

Beckum, I. (2010). A case study of teachers' perceptions of change and change implementation at a rural public 
school. (Doctoral dissertation), Walden University.

Boohene, R., y Williams A. A. (2012). Resistance to organizational change: A case study of Oti Yeboah Complex Limited. International Business and Management, 4(1), 135-145.

Boyce, M. E. (2003). Organizational learning is essential to achieving and sustaining change in higher education. Innovative Higher Education, 28(2), 119-136.

Brouwers, A., Tomic, W., y Boluijt, H. (2011). Job demands, job control, social support and self-efficacy beliefs as determinants of burnout among physical education teachers. Europe's Journal of Psychology, 7(1), 17-39. doi: 10.5964/ejop.v7i1.103.

Brown, C. G. (2012). A systematic review of the relationship between self-efficacy and burnout in teachers. Educational \& Child Psychology, 29, 47-63.

Bümen, N. T. (2010). Relationship between demographics, self-efficacy, and burnout among teachers. Demografik Değişkenler, Öğretmenlerin Özyeterlik ve Tükenmişlik Düzeyleri Arasındaki İlişkiler, 40, 16-35.

Campos, C. H., y Ramírez, S. M. (2018). Las TIC en los procesos educativos de un centro público de investigación. Apertura, 10(1), 56-70. doi: 10.18381/ Ap.v10n1.1160.

Caruth, G., y Caruth, D. (2013). Understanding resistance to change: a challenge for universities. Turkish Online Journal of Distance Education. 14(2), 1221.

Clarke, J. S., Ellett, C. D., Bateman, J. M., y Rugutt, J. K. (1996). Faculty receptivity/resistance to change, personal and organizational efficacy, decision deprivation and effectiveness in research I universities. Proceedings of the Association for the Study of Higher Education Meeting, 21. Congreso llevado a cabo en Menphis, EEUU.

Constantinescu \& Alexandrache (2014). Resistance to changes in the field of education. Procedia Social and Behavioral Sciences, 137, 70-73.

Córica, J. L. (2019). Estudio de la resistencia docente al cambio y a la incorporación de TIC en Argentina a través de un modelo de ecuaciones estructurales. (Tesis doctoral). Universidad Nacional de Educación a Distancia.

Cué, M., Díaz, G., Díaz, A., y Valdéz, M (2008). El artículo de revisión. Revista Cubana de Salud Pública 34(4). Recuperado de http://scielo.sld.cu/ scielo.php?script $=$ sci arttext\&pi$\mathrm{d}=$ So864-34662008000400011

D'Elia, S. (2019). Everyday enactments of resistance: portraits of secondary public school teachers navigating new professionalim. (Tesis doctoral). Montclair State University.

Devos, G., Buelens, M., y Bouckenooghe, D. (2007). Contribution of content, context, and process to understanding openness to organizational change: Two experimental simulation studies. The Journal of Social Psychology, 147(6), 607-629.

DuFour, R., y Marzano, R. J. (2011). Leaders of learning: How district, school, and classroom leaders improve student achievement. Solution Tree Press.

DuFour, R., DuFour, R., y Eaker, R. (2008). Revisiting professional learning communities that work: New insights for improving schools. Solution Tree.

Evers, W. J. G., Brouwers, A., y Tomic, W. (2002). Burnout and self-efficacy: A study on teachers' beliefs when implementing an innovative educational system in the Netherlands. British Journal of Educational Psychology, 72, 227-243.

Flamholtz, E., y Randle, Y. (2008). Leading strategic change: Bridging theory and practice. Cambridge University Press.

Folkers, D. A. (2005). Competing in the marketspace: Incorporating online education into higher education - An organizational perspective. Information 
Resources Management Journal, 18(1), 61-77.

Ford, J. D., y Ford, L. W. (2009a). Four conversations: Daily communication that gets results. Berrett-Koehler Publishers, Inc.

Ford, J. D., y Ford, L. W. (2009b). Resistance to change: A reexamination and extension. Research in Organizational Change and Development, 17, 211-239.

Ford, J. D., y Ford, L. W. (2010). Stop blaming resistance to change and start using it. Organizational Dynamics, 39(1), 24-36.

Gibson, S., y Dembo, M. H. (1984). Teacher efficacy: A construct validation. Journal of Educational Psychology, 76, 569-582.

Girao, G. S. (2015). Utilidad y tipo de revisión de literatura. Ene. 9(2) doi: 10.4321/ S1988-348X2015000200002

Gonsiewski, M. (2014). Resistance to change. Teacher variables that may influence the acceptance. (Master Thesis). Graduate School Appalachian State University.

Graetz, F., Rimmer, M., Lawrence, A., y Smith, A. (2002). Managing organizational change. John Wiley and Sons Ltd.

Grant, K. (2003). Making sense of education change at Thistle College: The existence of witchcraft, witches and shamans. International Journal of Educational Management, 17(2), 71-83.

Hargreaves, A. (2005). Educational change takes ages: Life, career, and generational factors in teachers' emotional responses to educational change. Teaching and Teacher Education, 21, 967-983.

Hartley, M. (2009). Leading grassroots change in the academy: Strategic and ideological adaptation in the civic engagement movement. Journal of Change Management, 9(3), 323-338.

Huberman, M. (1988). Teacher careers and school improvement. Journal of Curriculum Studies, 2O(2), 119-132.

Ibrahim, A., Al-Kaabi, A., y El-Zaatrari, W. (2013). Teacher resistance to educational change in the United Arab Emirates. International Journal of Research Studies in Education, 2(3), 25-36.

Kavanagh, M. H., y Ashkanasy, N. M. (2006). The impact of leadership and change management strategy on organizational culture and individual acceptance of change during a merger. British Journal of Management, 17, 81-103.

Keenan, K. M., y Marchel, M. A. (2007). Navigating change in higher education: The partnership experience of department faculty with an organization development consultant. Organization Development Journal, 25(1), 56-69.

Kezar, A. (2001). Understanding and facilitating organizational change: Recent research and conceptualizations. ASHEERIC Higher Education Report, 28(4), 1-10.

Kezar, A., y Eckel, P. (2002). The effect of institutional culture on change strategies in higher education. The Journal of Higher Education, 73(4), 435-460.

Kezar, A. (2005). Consequences of radical change in governance: A grounded theory approach. Journal of Higher Education, 76(6), 634-668.

Kotter, J., y Schlesinger, L. (1979). Choosing strategies for change. Harvard Business Review, 57, 106-114.

Kreitner, R., y Kinicki, A. (2010). Organizational behavior (9a. edition). McGraw-Hill Irwin.

Lane, I. F. (2007). Change in higher education: Understanding and responding to individual and organizational resistance. Journal of Veterinary Medical Education, 34(2), 85-92.

Martin, A. J., Jones, E. S., y Callan, V. J. (2005). The role of psychological climate in facilitating employee adjustment during organizational change. European Journal of Work and Organizational Psychology, 14(3), 263-89.

McBride, K. (2010). Leadership in higher education: Handling faculty resistance to 
technology through strategic planning. Academic Leadership, 8(4), 41.

McGregor, J. (2002). Flexible scheduling: How does a principal facilitate implementation? School Libraries Worldwide, 8(1), 71-84.

Merino-Trujillo, A. (2011). Como escribir documentos científicos (Parte 3). Artículo de revisión. Salud en Tabasco, 17(1-2), 3640. Recuperado de https://www.redalyc. org/articulo.oa?id=487/48721182006

Molina, P. L., Mesa, J., y Fredy, Y. (2018). Las TIC en Escuelas Rurales: realidades y proyección para la Integración. Praxis \& Saber, 9(21), 75-98. doi: 10.19053/22160159.v9.n21.2018.8924.

Padilla, P. S. (2018). Usos y actitudes de los formadores de docentes ante las TIC. Entre lo recomendable y la realidad de las aulas. Apertura, 1O(1), 132-148. doi: 688/68855405009.

Palmer, I., Dunford, R., y Akin, G. (2009). Managing organizational change: A multiple perspectives approach. McGraw-Hill Education.

Payne, C. M., y Kaba, M. (2007). So much reform, so little change: Building-level obstacles to urban school reform. Social Policy, 37(3/4), 30-37.

Per, D. (2004). School development: Theories and strategies. Continuum International USA Publishing Group.

Rayan, S., y Ackerman, D. (2005). Using pressure and support to create a qualified workforce. Education Policy Analysis Archives, 13(23), 1-19.

Reilley, W. (1989). Understanding that resistance to change is inevitable. Managing change in higher education, 5 , 53-66.

Ryals, K. N. (2019). Teacher resistance and change: a mixed method case study examination of faculty perceptions of professional learning communities in supporting teacher change and student achievement in a well-resourced, high achieving secondary school. (Tesis doctoral). Widener University, Partial Fulfillment.

Silcox, S., MacNeill, N., y Cavanagh, R. (2003). Principal leadership and school renewal. Paper presented at the 2003 Annual Conference for the Australian Association for Research in Education. Auckland. Recuperado de http://aare. edu.au/o3pap/silo3419.pdf

Snyder, R. (2017). Resistance to Change among Veteran Teachers: ProvidingVoice for More Effective Engagement. International Jounal of Educational Leadership Preparation, 12(1), 1-14.

Tyack, D., y Cuban, L. (1995). Tinkering toward utopia: A century of public school reform. Harvard University Press.

Yılmaz, D., y Kılıçoğlu, G. (2013). Resistance to change and ways of reducing resistance in educational organizations. European Journal of Research on Education. 1(1), 14-21.

\section{PERFIL ACADÉMICO Y PROFESIONAL DEL AUTOR}

José Luis Córica. Doctor Internacional en Educación de la Universidad Nacional de Educación a Distancia. Magister en Enseñanza y Aprendizajes Abiertos y a Distancia, Licenciado en Análisis de Sistemas, Licenciado en Comunicación Corporativa y Licenciado en Gestión de Empresas. Director de la Fundación Latinoamericana para la Educación a Distancia. Sus temas de interés son educación y tecnologías, innovación tecnológica institucional y analítica educativa.

E-mail: jlcorica@hotmail.com, jlcorica@flead.org 
Dirección:

Fundación Latinoamericana

para la Educación a Distancia

Coronel Olascoaga 2053 Oficina 1 (5500)

Mendoza, Argentina

Fecha de recepción del artículo: 27/01/2020

Fecha de aceptación del artículo: 08/02/2020

Fecha de aprobación para maquetación: 15/04/2020 